\title{
Nuclear symmetry energy in calcium-calcium collisions (INDRA-VAMOS)
}

\author{
P.C. WigG ${ }^{1}$, M. Boisjoli ${ }^{3}$, M. Chartier ${ }^{1}$, A. ChBihi ${ }^{3}$, R. Lemmon $^{2}$, \\ J.D. Frankland ${ }^{3}$, N. Le Neindre ${ }^{4}$, and P. Marini ${ }^{3}$ \\ for the INDRA-VAMOS E503 COLLABORATION
}

${ }^{1}$ Department of Physics, University of Liverpool, L69 7ZE, UK 2 STFC Daresbury Laboratory, Warrington, Cheshire, WA4 4AD, UK

3 GANIL, CEA, IN2P3-CNRS, B.P.5027, F-14021 Caen Cedex, France ${ }^{4}$ LPC. IN2P3-CNRS. ENSICAEN et Université, Caen Cedex, FRANCE

\begin{abstract}
The density dependence of the symmetry energy is of great interest to many fields of nuclear physics and nuclear astro-physics. The E503 INDRA-VAMOS experiment performed at GANIL in 2007 is intended to provide further sub-saturation constraints using calcium-calcium collisions around the Fermi energy $(35 \mathrm{AMeV})$. In these proceedings this experiment will be discussed in the context of the physics it is aiming to study and will give a brief summary of the current progress of the data analysis.
\end{abstract}

\section{Background information}

The nuclear equation of state relates the energy of a nuclear system to its temperature, density, pressure and isospin. For symmetric matter this relation has been well studied in the past but its behaviour for asymmetric matter has comparatively little constraint.

In the first approximation if we take an expansion around the saturation density of nuclear matter $\left(\rho=\rho_{0} \simeq 0.16 \mathrm{fm}^{-3}\right)$ we have:

This is an Open Access article distributed under the terms of the Creative Commons Attribution License 2.0, which permits unrestricted use, distribution, and reproduction in any medium, provided the original work is properly cited. 


$$
E(\rho, \delta)=E(\rho, \delta=0)+E_{\text {sym }}(\rho) \cdot \delta^{2}+\ldots
$$

Where $\delta=\frac{\rho_{n}-\rho_{p}}{\rho}$.

If we ignore the higher order terms in this expansion and keep the first two (the parabolic approximation) then we see that the first part is symmetric, having no dependence on $\delta$ and the second term is asymmetric, proportional to $\delta^{2}$. One should note two things concerning the asymmetric term. The first is its dependence upon $\delta^{2}$ which renders it much weaker and therefore more difficult to access than the symmetric term (as $\delta$ is normalised). The second point is that it contains a density dependence $E_{\text {sym }}(\rho)$. This density dependence, "the density dependence of the symmetry energy" is of considerable interest in many fields of nuclear physics and nuclear astrophysics:

- Heavy Ion Collisions dynamics

- Iso-vector collective vibrations: Pygmy Dipole Resonances, Giant Dipole Resonances

- Neutron star physics: Mass-Radius relation, crustal physics, cooling rates, ...

- Neutron skin thickness

- Location of the drip line

The current understanding of $E_{\text {sym }}(\rho)$ can be summarised by the following plots. For fig 1. you can see a plot of some frequently used parametrisations of $E_{\text {sym }}(\rho)[1]$ and in fig 2. there is a summary diagram demonstrating the current level of constraint in the sub-saturation region [2].

It is clear from fig 1 that these parametrisations are better constrained in the sub-saturation region $\left(\rho / \rho_{0}<1\right)$ than they are in the super-saturation region. From this point on however the focus will remain on the subsaturation region, as it is this region we are concerned with in E503 - one of its primary targets is to further constrain the sub-saturation dependence of $E_{\text {sym }}(\rho)$.

It should also be apparent from fig 2 that while there are already constraints in the sub-saturation region, there is still a lot of freedom allowed for individual parameters and this will feed through into larger uncertainties on any conclusions drawn from them. 


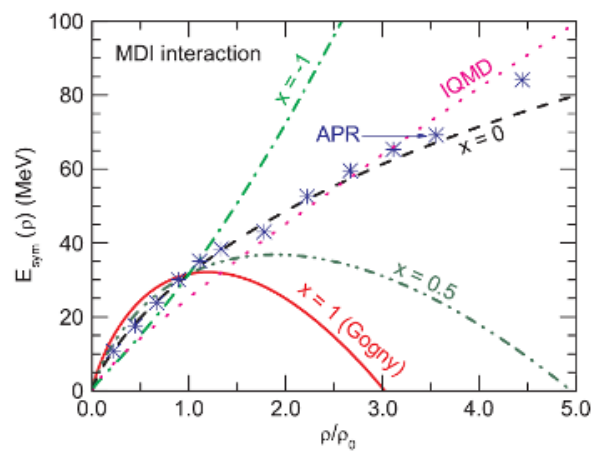

Figure 1: Common parametrisations of $E_{\text {sym }}(\rho)$ (Colour online) [1].

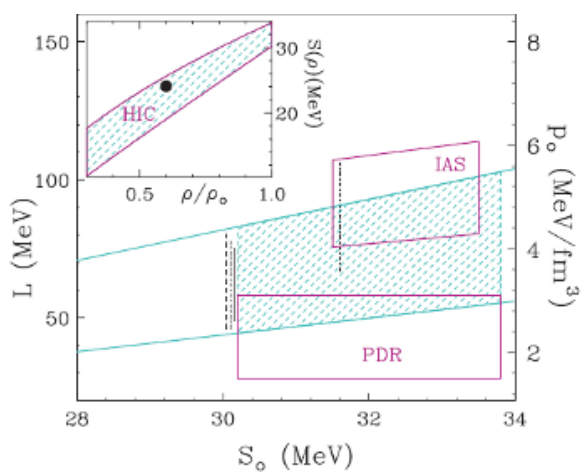

Figure 2: Current constraints (Colour online) [2].

To be able to constrain $E_{\text {sym }}(\rho)$ in this way we need to find a set of observables that can access the symmetry energy and which will preferably reduce contributions coming from unwanted effects that we wish to exclude. There are several possible observables that exist for the study of $E_{\text {sym }}(\rho)$ in the sub-saturation region at the moment, and a few of them will be mentioned briefly here.

\section{$1.1 \quad$ Iso-scaling parameters}

The first observable is that of the iso-scaling parameters. In this case we make use of the phenomena of iso-scaling in which the yield ratio of a given isotope produced in two different fragmenting systems [6] (denoted as 1, 2) follows the observed relation: 


$$
R_{21}=C \cdot \exp (\alpha N+\beta Z)
$$

Where $\alpha$ and $\beta$ are the iso-scaling parameters for neutrons and protons respectively: $\alpha=\left(\mu_{n(2)}-\mu_{n(1)}\right) / T$ and $\beta=\left(\mu_{p(2)}-\mu_{p(1)}\right) / T$ if the two systems are at the same temperature. Where $\mu$ denotes the chemical potential of the nucleon in question and $T$ the temperature.

It is not immediately apparent from the above relation how one can access the symmetry energy with this observable but it emerges from considerations of the nuclear free energy $G(N, Z)$ that the $\alpha$ parameter depends only on the symmetry energy term, and can therefore be used to access information about the symmetry energy.

The mirror yield ratio $R_{7}$ is also useful when looking at these isotopic distributions as it reduces the mass dependency of the symmetry energy:

$$
R_{7}=\ln \left(Y\left({ }^{7} L i\right) / Y\left({ }^{7} B e\right)\right)
$$

\subsection{Neutron-proton double ratio}

Another observable which can be used to access $E_{\text {sym }}(\rho)$ is the neutronproton double ratio. In this observable we construct the double ratio from the neutron and proton differential multiplicity spectra (with respect to the centre of mass) the advantage in doing this is that it reduces the effect of measurement bias between the neutrons and protons in the data. This observable gets its sensitivity to the symmetry energy from the fact that neutrons and protons have opposing signs for their symmetry potentials.

$$
\frac{d M_{n}(A) / d E_{c . m}}{d M_{p}(A) / d E_{c . m}} \cdot \frac{d M_{p}(B) / d E_{c . m}}{d M_{n}(B) / d E_{c . m}}
$$

\subsection{Isospin transport ratio}

The isospin transport ratio is the last experimental observable that will be mentioned. The goal of this observable is to measure the degree of isospin transport (equilibration) that occurs while minimising unwanted effects coming from pre-equilibrium emission, sequential decay and from Coulomb interactions. To do this the ITR exploits the fact that these effects are present in both the symmetric and asymmetric reaction systems to approximately the same degree, if the collision energy is the same:

$$
R=\frac{2 X^{A+B}-X^{A+A}-X^{B+B}}{X^{A+A}-X^{B+B}}
$$


Here $X$ represents any observable with a linear dependence on the asymmetry of the projectile-like fragment $\delta_{P L F}$, for example $\alpha, R_{7} . A$ and $B$ denote the two reaction systems, one neutron-rich, the other neutron-poor.

\section{Experimental setup}

Both this experiment (E503) and its sister experiment (E494s) make use of the same experimental setup, namely, the INDRA- $4 \pi$ charged particle multi-detector array coupled to the VAMOS magnetic spectrometer (see fig 3.) INDRA is a $4 \pi$ array of multi-stage telescopes composed of Ionisation Chambers, Silicon and Caesium Iodide detectors set into 17 rings (14 rings for these experiments as the first 3 rings were removed to allow coupling to the magnetic spectrometer) with the exact telescope composition being a function of the ring number [3]. VAMOS is a large acceptance magnetic spectrometer with a focal plane detection system composed of an Ionisation Chamber, a Silicon detector array and a Caesium Iodide wall $[4,5]$.

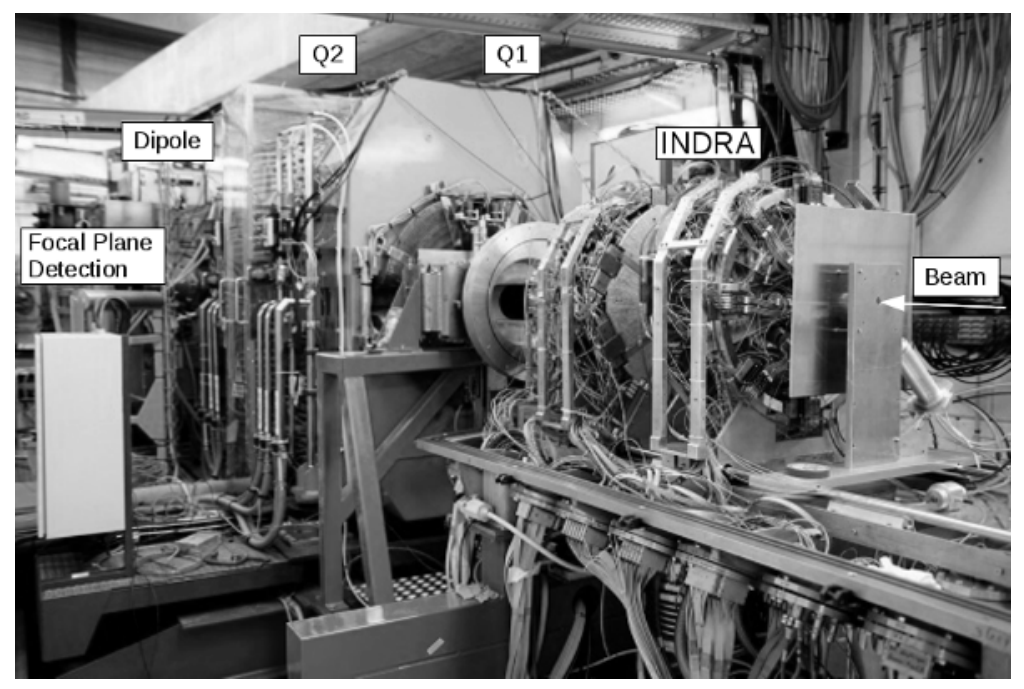

Figure 3: INDRA-VAMOS Coupling.

This experimental setup is crucial as it allows direct measurement of both the projectile-like residue in VAMOS and the associated light charged particles in INDRA. Therefore any conclusions drawn from the data can be made with a great reduction in the degree of assumption that would otherwise be necessary if either of the aforementioned detectors were not present. 


\section{PID_INDRA SI_CSI (Ring 5 Module 3)}

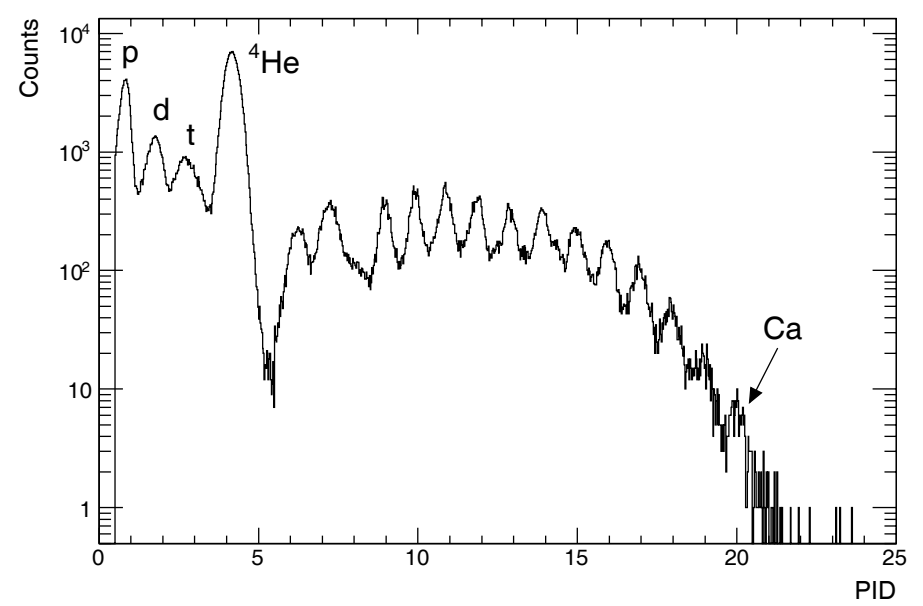

Figure 4: PID plot (see text) from INDRA Si-CsI identification.

\section{Status of detector calibration}

Before the data can be analysed for physics it is necessary to have a good calibration for both INDRA and VAMOS detectors. Once this calibration

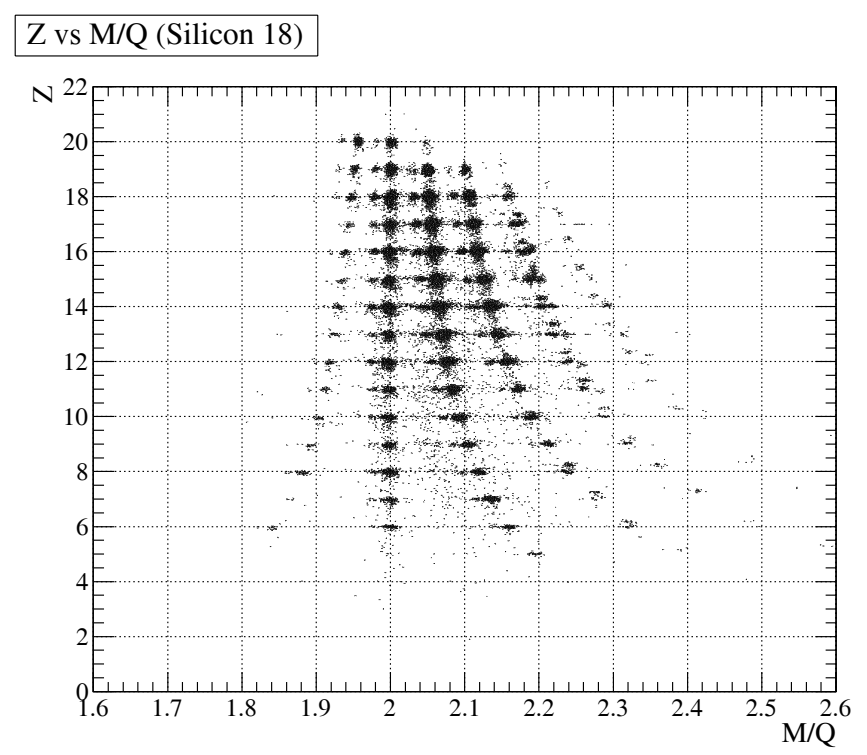

Figure 5: Example Z vs. M/Q plot for VAMOS. 
is done the resulting particle identification from both detectors must be combined into a single physics data set so that the analysis can be performed using the full phase-space coverage for each event.

To demonstrate the results of the identification procedures already in place for INDRA an example particle identification plot has been included (not full statistics, just a few runs of data) where the parameter $P I D=$ $Z+0.1 \cdot(A-2 Z)$. In this plot everything below $Z=8$ is identified in both $A$ and $Z$, beyond this point the identification is made only in $Z$ - this isotopic identification threshold will be set module-by-module. In general we can expect to get a mass identification for isotopes up to Boron, however we see in some modules isotopic resolution up to Oxygen. Notice that the $Z$ identification runs right up to the $Z$ of the beam. The isotopic separation we have in VAMOS is also well demonstrated in fig 5.

At this point in time both INDRA and VAMOS have been effectively calibrated, although there is still some fine tuning and testing to be done before the data from each multi-detector system can be combined and analysed with confidence.

\section{Conclusion}

In summary, the E503 INDRA-VAMOS experiment represents a real opportunity to explore the sub-saturation density dependence of the symmetry energy by coupling a $4 \pi$ charged particle multi-detector to a high resolution magnetic spectrometer. At this point both INDRA and VAMOS data sets are on the threshold of being combined to give the full phase space coverage for each event and it is hoped that the physics analysis will begin very soon.

\section{References}

[1] B-A Li, L-W Chen \& C.M. Ko, Physics Reports, (2008) 464, 113

[2] M.B. Tsang et al. arXiv:1101.3648v1 [nucl-ex] (2011)

[3] J. Pouthas et al. Nucl Instrum Meth A 357 (1995) 418-442

[4] S. Pullanhiotan et al. Nucl Instrum Meth A 593 (2008) 343- 352

[5] S. Pullanhiotan et al. Nucl Instrum Meth B 266 (2008) 4148-4152

[6] C. Dorso, Phys. Rev. C 73 (2006) 034605. 